\title{
ON INNER-OUTER AND SPECTRAL FACTORIZATIONS
}

\author{
Cheng-Chih Chu John C. Doyle
}

Honeywell Inc., Systems and Research Center, Minneapolis, Minnesota

\section{Abstract}

This paper outlines methods for computing the key factorizations necessary to solve general $H_{2}$ and $H_{\infty}$ linear optimal control problems.

\section{Notation}

$L_{a} \triangleq\{$ Lebesque space $\}$

$H_{\alpha} \triangleq\{$ Hardy space

$R \triangleq\{$ Proper, real-rational\}

$R^{p \times m} \triangleq\{p \times m$ matrices in $R\}$ (similarly for $H$ and $L$ )

$\left.\mid \begin{array}{l|l}A & B \\ \hline C & D\end{array}\right] \triangleq D+C(s I-A)^{-1} B$

$r \subset f \triangleq$ right coprime factorization over $R H_{\infty}$

Throughout this paper, $\alpha$ will be used whenever either $\alpha=2$ or $\alpha=\infty$ would apply equally. The term unit in $R H_{\infty}$ refers to any

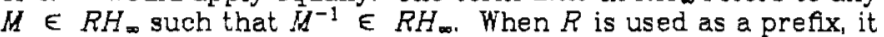
denotes real-rational.

\section{Introduction :}

The importance of inner-outer (IOF), spectral and coprime factorizations in obtaining solutions to certain $H_{2}$ and $H_{\infty}$ optimal control problems has been known for some time. The solution to the general $H_{a}(\alpha=2, \infty)$ optimal control problems $[1],[2]$ uses these factorizations and, in addition, the "complementary inner factor" (CIF), to reduce the general problem to that of approximating an $L_{a}$ rational matrix by one in $H_{a}$.

This paper focuses on the factorizations used in [2] and in particular, on explicit formulas and methods for computation. We show that all the factorizations needed in the $H_{2}$ and $H_{\text {. }}$ optimal synthesis problem can be obtained using standard real matrix operations on state-space representations. The Algebraic Riccati Equation (ARE) plays a central role in computing the desired factorizations. Because of space limitations, the "proofs" of the results in this paper are extremely sketchy.

\section{Background :}

The general $H_{a}$ optimal control problem is shown in the following figure.

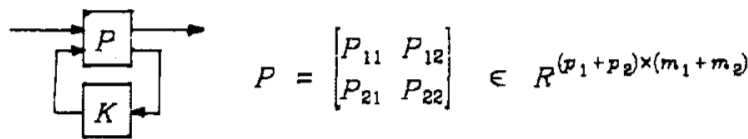

The objective is to find a stabilizing $K \in R^{m^{m} p_{2}}$ which solves $\min _{K}|| \mathbf{F}_{l}(P ; K) \mid l a$ where $\mathbf{F}_{l}(P ; K) \triangleq P_{11}+P_{12} K\left(I-P_{22} K\right)^{-1} P_{21}$. For nontriviality, assume that $p_{1}>m_{2}$ and $m_{1}>p_{2}$.

The first step is to find $K_{0}=\left[\begin{array}{ll}K_{11} & K_{12} \\ K_{21} & K_{22}\end{array}\right] \in R^{\left(m_{2}+p_{2}\right) \times\left(p_{2}+m_{2}\right)}$ such that $\mathbf{F}_{l}\left(P ; F_{l}\left(K_{0} ; Q\right)\right)=F_{l}(T ; Q)=T_{11}+N Q \tilde{N} \in R H_{a}^{p_{1} \times m_{1}}$ is

* This work has been supported by Honeywell Internal Research and Development Funding, the Office of Naval Research under ONR Research Grant N00014-82-C-015\%, and the U.S. Air Force of Scientific Research Grant F49620-82C-0090. stable and affine for any $Q \in R H_{\infty}^{m} 2^{x_{p}}$. This is the Youla parametrization of all stabilizing controllers and is obtained by finding coprime factorizations of $\mathrm{P}$ over the ring of stable rationals and solving a double Bezout identity to obtain the coefficients of $K_{0}$.

We are interested in a particular $K_{0}$ which results in both $N$ and $\tilde{N}$ being inner. That is, $N^{*} N=I$ and $N N^{*}=I$. This requires a coprime factorization with inner numerator. In addition, we require $N_{\perp}$ and $\tilde{N}_{\perp}$ inner so that $\left[N N_{\perp}\right]$ and $\left[\begin{array}{|}\tilde{N}_{\perp} \\ \tilde{N}_{1}\end{array}\right]$ are square and inner. $N_{\perp}$ and $\tilde{N}_{\perp}$ are called complementary inner factors (CIF). With these we have that

$$
\begin{aligned}
\left|T_{11}+N Q \tilde{N}\right|_{a} & =\left|\left[N N_{1}\right]^{*}\left[T_{11}+N Q \tilde{N}\right]\right|\left[\begin{array}{c}
\tilde{N} \\
\tilde{N}_{1}
\end{array}\right]^{*} \|_{a} \\
& =\|\left[\begin{array}{ll}
N & N_{1}
\end{array}\right]^{*}\left[T_{11}\right]\left[\begin{array}{l}
\tilde{N} \\
\tilde{N}_{1}
\end{array}\right]^{*}+\left.\left[\begin{array}{ll}
Q & 0 \\
0 & 0
\end{array}\right]\right|_{a}
\end{aligned}
$$

since both the $\alpha=2$ and $\infty$ norms are unitary invariant.

The $H_{2}$ case immediately reduces to a best approximation problem with $Q_{\text {opt }}=\mathbf{P}_{H_{\mathrm{g}}}\left(N^{*}\left[T_{11}\right] N^{*}\right)$, where $\mathbf{P}_{H_{2}}$ denotes projection onto $H_{2}$. The $H_{\text {so }}$ case is somewhat more complicated and requires an additional spectral factorization. To see how this arises, consider the spegial case when $T_{11} \tilde{N}_{\perp}^{*}=0$ and (2.1) reduces to $G=N_{\perp}^{*}\left[T_{11}\right] \tilde{N}^{*}$.

It is easily verified that for any $\gamma>\|G\|_{\infty}$

$$
\|\left[\begin{array}{c}
R+Q \\
G
\end{array}\right]||_{\infty} \leq \gamma \text { iff } \quad\left|\left(\gamma^{2} I-G^{*} G\right)^{-k}(R+Q)\right|_{\infty} \leq 1
$$

where $(H)^{*}$ denotes the unit spectral factor of the paraHermitian matrix $H$. Thus, the $H_{\infty}$ problem also reduces to a best approximation problem since the $\left(\gamma^{2} I-G^{*} G\right)^{-\pi}$ is a unit and can be absorbed into $Q$. The general case similarly involves both inner-outer and spectral factorizations [2]. The remainder of the paper outlines methods for computing these factorizations.

\section{Algebraic Riccati Equation :}

Consider the Algebraic Riccati Equation,

$$
F^{T} X+X F-X W X+Q=0
$$

where $F, W, Q \in \mathbb{R}^{n \times n}, W=W^{T} \geq 0$ and $Q=Q^{T}$ with the associated Hamiltonian matrix

$$
A_{H}=\left[\begin{array}{cc}
F & -F \\
-Q & -F^{T}
\end{array}\right]
$$

Our main interest is to find the unique real symmetric stabilizing solution such that the matrix $(F-W X)$ is asymptotically stable. For simplicity we will use "solution" of the ARE to mean a real symmetric one. The ARE considered here is more general than the ARE which arises in linear quadratic optimal control and Kalman-Bucy flltering theory in that there is no assumption on the definiteness of the matrix $Q$.

The following theorem gives the necessary and sufficient conditions for the existence of a unique stabilizing solution of (ARE). Without loss of generality, we will assume that $W=G G^{T}$. This is a slight generalization of a theorem of Kucera [3]. 


\section{Theorem 3-1:}

The stabilizability of $(F, G)$ and $\operatorname{Re}\left[\lambda_{i}\left(A_{H}\right)\right] \neq 0$

$(\forall i=1,2, \ldots \ldots, 2 n)$ are necessary as well as sufficient for the existence of a unique stabilizing solution of (ARE).

Remarks :

(1) The unique stabilizing solution of Theorem 3-1 will be denoted by Ric $\left(A_{H}\right)$

(2) If $Q \geq 0$, then the stabilizing solution $X \geq 0$.

Theorem 3-2:

If $Q=H^{T} H \geq 0$ in (ARE) and $X$ is its solution, then $\operatorname{Ker}(X)=\operatorname{Ker}(H)$.

\section{Inner-Outer Factorization (IOF) :}

Assume $G=\left[\frac{A \mid B}{C \mid D} \mid \in R^{p \times m} \quad(p \geq m)\right.$ and the realization is minimal. We use the notation. " $D_{1}$ " for the orthogonal complement of $D$ and $R^{*}(R \geq 0)$ for any square matrix $V$ s.t. $V^{T} V=R$.

Theorem 4-1:

$G$ has a $r \subset f G=N H^{-1}$ with $N$ inner if and only if $G$ has no (transmission) zeros on the $j$ w-axis, including $\propto$.

A particular realization for the factorization is

$\left[\begin{array}{l}H \\ N\end{array}\right]=\left[\begin{array}{c|c}A-B F & B R^{-\not h} \\ \hline-F & R^{-\not h} \\ C-D F & D R^{-\not h}\end{array}\right] \in R H_{\infty}^{(m+p) \times m}$

where $R=D^{T} D>0, F=R^{-1}\left(B^{T} X-D^{T} C\right)$

and $X=\operatorname{Ric}\left[\begin{array}{cc}A-B R^{-1} D^{T} C & -B R^{-1} B^{T} \\ -C^{T} D_{1} D_{1}^{T} C & -\left(A-B R^{-1} D^{T} C\right)^{T}\end{array}\right] \geq 0$

[Proof] :

(only if) :

Suppose $G=N H^{-1}$ is a rof and $N^{*} N=I$, Then $G^{*} G=\left(H^{-1}\right)^{*} H^{-1}>0$ on the $j \omega$-axis since $M$ is stable. Thus $G$ has no $j$ - waxis zeros.

(if) :

The if part can be proven by directly verifying that the above realization is a $r \circ f$ of $G$ and that $N^{*} N=I$. The conditions on $\mathrm{G}$ insure, by Theorem 3-1, the existence of $X$.

\section{Theorem 4-2 :}

If $p>m$ in Theorem 4-1, then there exists a CIF $N_{\perp} \stackrel{f}{\epsilon} H_{\infty} p \times(p-m)$ such that the matrix $\left[N N_{\perp}\right]$ is square and inner. A particular realization is

$$
N_{\perp}=\left[\begin{array}{c|c}
A-B F & -X^{\dagger} C^{T} D_{1} \\
\hline C-D F & D_{\perp}
\end{array}\right]
$$

where $X^{\dagger}$ is the pseudo-inverse of the stabilizing Riccati solution $X$ from Theorem 4-1.

[Proof]:

The proof consists of verifying directly that $\left[N N_{1}\right]$ is inner using the above realization for $N_{1}$ and the realization for $N$ from Theorem 4-1. A key step requires that $\operatorname{Ker}(X) \subset \operatorname{Ker}\left(D^{T} C\right)$, which follows from Theorem 3-2.

\section{Theorem 4-3:}

There exists a rof $G=N M^{-1}$ such that $M \in R H_{\infty}^{m \times m}$ is inner if and only if $G$ has no poles on the $j \omega$-axis. A particular realization is

$$
\left[\begin{array}{l}
M \\
N
\end{array}\right]=\left[\begin{array}{c|c}
A-B F & B \\
\hline-F & I \\
C-D F & D
\end{array}\right] \in R H_{\infty}^{(m+p) \times m}
$$

where $F=B^{T} X$ and $X=\operatorname{Ric}\left[\begin{array}{cc}A & -B B^{T} \\ 0 & -A^{T}\end{array}\right] \geq 0$.

[Proof]:

Similar to Theorem 4-1.
Remarks :

(1) The minimality condition in Theorem 4-3 can be weakened to $(A, B)$ stabilizable and $\operatorname{Re}\left[\lambda_{i}(A)\right] \neq 0$ and the theorem still holds,

(2) If $G \in R H_{\infty}^{p \times m}$ in Theorem 4-1, ther $M$ is a unit in $R H_{\infty}$. In this case, $G \square N\left(M^{-1}\right)$ is called "inner-outer factorization" (IOF).

(3) Dual results for all factorizations can be obtained when $p \leq m$. In these factorizations, output injection using the dual Riccati solution replaces state feedback to obtain corresponding left factorizations.

\section{Spectral Factorization :}

Recall from Section 2 that the $H_{\infty}$ solution requires the computation of the spectral factor which is a unit in $R H_{\infty}$. The following theorem characterizes this spectral factor. The same assumptions on $G$ apply here as did in Section 4 except that $G \in R L_{\infty}^{P_{\infty} \times m}$.

\section{Theorem 5-1:}

If $\gamma>G(s)=$ there exists a unit $M \in R H_{\infty}^{m \times m}$ such that $H^{*} H=\gamma^{2} I-G^{*} G$. A particular realization is given by

$$
M=\left[\begin{array}{l|l}
A-K_{F} K_{C} & B-K_{F} \\
\hline R^{H} K_{C} & R^{H /}
\end{array}\right]
$$

where $R=\gamma^{2} T-D^{T} D>0, K_{C}=R^{-1}\left(B^{T} X-D^{T} C\right), K_{F}=Y K_{C}{ }^{T} R$

$$
X=\operatorname{Ric}\left[\begin{array}{cc}
A+B R^{-1} D^{T} C & -B R^{-1} B^{T} \\
C^{T}\left(I+D R^{-1} D^{T}\right) C & -\left(A+B R^{-1} D^{T} C\right)^{T}
\end{array}\right]
$$

and

$$
Y=\operatorname{Ric}\left[\begin{array}{cc}
A^{T} & -K_{C}{ }^{T} R K_{C} \\
0 & -A
\end{array}\right]
$$

[Proof] :

The proof consists of verifying from the above realizations that $M$ and $M^{-1}$ are stable and that $M^{*} H=\gamma^{2} I-G^{*} G$

Remark :

If $G$ is stable, then $Y=0$ and $K_{F}=0$.

\section{Implementation :}

All the factorizations in the theorems have explicit statespace representations. Thus, the implementation of algorithms can be done easily using real matrix operations. Two key steps in the algorithms are:

(1) Solution of ARE : the ARE can be solved using the Schur method which reduces the Hamiltonian matrix to rea Schur form by orthogonal similarity transformation and then uses the invariant subspace associated with $n$ stable eigenvalues to find the stabilizing solution. See [4] for a complete treatment.

(2) $\left(D^{T} D\right)^{k / 2}$ and $D_{\perp p}$; using the QR factorization, we find $D=\left[\begin{array}{ll}Q_{1} & Q_{2}\end{array}\right]\left[\begin{array}{l}R \\ 0\end{array}\right]$ where $Q=\left[\begin{array}{lll}Q_{1} & Q_{2}\end{array}\right]$ is orthogonal. Then $R=\left(D^{T} D\right)^{x}$ and $D_{1}=Q_{2}$.

Using the well-known software packages EISPACK, LINPACK and some of their extensions, experimental versions of these algorithms have been implemented successfully. Experience to date indicates that these algorithms are very reliable.

\section{References}

[1] J. Doyle, "Synthesis of Robust Controllers and Filters," Proceedings of Z2nd Conference on Decision and Control, December, 1983.

[2] J. Doyle, Lectures Notes, ONR/Honeyuell Workshop on Advances in Multivariable Control, October 8-10,1984, Minneapolis, Minnesota.

[3] V. Kứera, "A Contribution to Matrix Quadratic Equations", IEEE Trans. on Auto. Control, vol. 17 pp. 344-347, June, 1972

[4] A. J. Laub, "A Schur Method for Solving Algebraic Riccati Equations." IEEE Trans, on Auto. Control, vol. 24 .pp. 913921, December, 1979 